PROCEEDINGS OF THE

AMERICAN MATHEMATICAL SOCIETY

Volume 125, Number 1, January 1997, Pages 245-250

S 0002-9939(97)03591-0

\title{
SEMICONSTANT MEASURES ON HYPERBOLIC LOGICS
}

\author{
MARJAN MATVEJCHUK
}

(Communicated by J. Marshall Ash)

\begin{abstract}
We characterize the set of all semiconstant measures on the hyperbolic logics of projections in indefinite metric spaces and describe the set of all probability measures on these logics.
\end{abstract}

\section{INTRODUCTION}

One of the basic problems related to the propositional calculus approach to the foundations of quantum mechanics [1] is the description of probability measures (called states in physical terminology) on the set of experimentally verifiable propositions regarding a physical system. The set of propositions form an orthomodular partially ordered set, where the order is induced by a relation of implication, and is called a quantum logic.

An important intepretation of a quantum logic is the set $\Pi$ of all orthogonal projections of a Hilbert space $H$. The remarkable Gleason theorem [2] asserts: Let $H$ be a Hilbert space, $\operatorname{dim} H \geq 3$ and let $\mu: \Pi \rightarrow R$ be a probability measure. There exists a positive trace class operator $T$ such that $\mu(p)=\operatorname{tr}(T p), \forall p \in \Pi$.

The problem of the construction of a quantum field theory leads to the indefinite metric spaces [3]. In this case, the set $\mathcal{P}$ of all $J$-orthogonal projections serves to be an analog to the logic $\Pi$. There is an indefinite analog to the Gleason theorem [4]. Let $H$ be a J-space, dim $\geq 3$ and let $\mu: \mathcal{P} \rightarrow R$ be an indefinite measure. There exist a $J$-selfadjoint trace class operator $T$ and a semiconstant measure $\mu_{0}$ such that $\mu(p)=\operatorname{tr}(T p)+\mu_{0}, \forall p \in \mathcal{P}$.

\section{Preliminary notions}

We present the necessary definitions and notations. Let $H$ be a space with an indefinite metric [...], a canonical decomposition $H=H^{+}[\dot{+}] H^{-}$, and a canonical symmetry $J$. Following the terminology of [5], $H$ is a Krein space (sometimes $H$ is called a $J$-space). $H$ is a Hilbert space with respect to the inner product (see [5]) $(z, y)=[J z, y]$. There exist orthogonal projections $P^{+}$and $P^{-}$such that $P^{+}+P^{-}=I, J=P^{+}-P^{-}$and $P^{+} H=H^{+}, P^{-} H=H^{-},[z, y]=(J z, y), \forall z, y \in$ $H$. Let $p \in B(H)$. It is easy to see that $[p z, y]=[z, p y], \forall z, y \in H \Leftrightarrow p=J p^{*} J$.

Received by the editors February 14, 1994 and, in revised form, July 13, 1995.

1991 Mathematics Subject Classification. Primary 81P10, 46L50, 46B09, 46C20, 03G12; Secondary 28A60.

Key words and phrases. Quantum logics, measure, indefinite metric space, $W^{*}$-algebra.

Supported by The Russian Foundation for Fundamental Research, grant no. 93-011-16099.

(C)1997 American Mathematical Society 
A vector $z \in H$ is said to be positive (negative) if $[z, z]>0([z, z]<0)$. The set $\Gamma \equiv\left\{f \in H:[f, f]^{2}=1\right\} \quad(=\{f \in H:[f, f]=1$ or $[f, f]=-1\})$ is an analog to the unit sphere $S=\{f \in H:(f, f)=1\}$. For any operator $p$ we denote by $e_{p}$ the orthogonal projection onto the subspace $\overline{p H}$.

A $W^{*}$-factor $\mathcal{A}$ in $H$ is called a $W^{*} J$-factor, if $J \in \mathcal{A}$. Let $\Pi$ be the set of all orthogonal projections in $\mathcal{A}$. Let $\mathcal{P}(=\mathcal{P}(\mathcal{A}))$ be the set of all $J$-selfadjoint projections in $\mathcal{A}$, i.e., $\mathcal{P}=\left\{p \in \mathcal{A}: p^{2}=p,[p z, y]=[z, p y], \forall z, y \in H\right\}$.

Denote by $\mathcal{P}^{+}\left(\mathcal{P}^{-}\right)$the set of all projections $p \in \mathcal{P}$, for which the subspace $p H$ is positive, i.e., $(\forall z \in p H, z \neq 0,[z, z]>0)$ (respectively, negative, i.e., $\forall z \in p H$, $z \neq 0,[z, z]<0)$. Any projection $e \in \mathcal{P}$ is representable in the form $e=e_{+}+e_{-}$, where $e_{+} \in \mathcal{P}^{+}, e_{-} \in \mathcal{P}^{-}$. Note that $p \in \mathcal{P}^{+} \Rightarrow p J \geq 0$ and $p \in \mathcal{P}^{-} \Rightarrow p J \leq 0$. Let $p$ be a projection. Then $p=e_{p} p=e_{p}+e_{p} p e_{p}^{\perp}$. Hence

$$
p p^{*}=e_{p}+e_{p} p e_{p}^{\perp} p^{*} e_{p} \geq e_{p} .
$$

Thus $\left(p p^{*}\right)^{1 / 2} \geq e_{p}^{1 / 2}=e_{p}$. Now it is clear that if $p \in \mathcal{P}^{+}$then $e_{p} \leq\left(p p^{*}\right)^{1 / 2}=$ $\left(p J J p^{*}\right)^{1 / 2}=(p J p J)^{1 / 2}=p J$ and if $p \in \mathcal{P}^{-}$then $e_{p} \leq-p J$. Let $x \in \mathcal{A}$ be such that $x \geq e_{x}$ and $e_{x} \leq P^{+}$, and let $v$ be an arbitrary isometry with the initial projection $e_{x}$ and the final one $v v^{*} \leq P^{-}$. Define an operator

$$
p=x+v\left(x^{2}-x\right)^{1 / 2}-\left(x^{2}-x\right)^{1 / 2} v^{*}-v\left(x-e_{x}\right) v^{*} .
$$

It can be easily verified that $p^{2}=p$ and $J p^{*} J=p$. Hence $p \in \mathcal{P}$. Since $p^{*} J p \geq 0$, it follows that $[p z, p z]=(J p z, p z)=\left(p^{*} J p z, z\right) \geq 0, \forall z \in H$. Thus $p \in \mathcal{P}^{+}$. (It can be proved [6] that any projectin $p \in \mathcal{P}^{+}$has the form (1) where $x=P^{+} p P^{+}$and $v$ is the partial isometry from the polar decomposition $P^{-} p P^{+}=v\left|P^{-} p P^{+}\right|$.) In order to emphasize the fact that $p$ from (1) depends on $x$ and $v$, we shall use the notation $p(x, v)$ as well.

A projection $p \in \mathcal{P}^{+}\left(p \in \mathcal{P}^{-}\right)$is said to be maximal positive (maximal negative) if the subspace $p H$ is the maximal positive (maximal negative) subspace. Let $\mathcal{P}_{m}^{+}$ $\left(\mathcal{P}_{m}^{-}\right)$be the set of all maximal positive (maximal negative) projections in $\mathcal{P}$. By [5, Theorem 45, §4], $p \in \mathcal{P}_{m}^{+} \Leftrightarrow p \in \mathcal{P}^{+}$and $P^{+} p H=H^{+}\left(p \in \mathcal{P}_{m}^{-} \Leftrightarrow p \in \mathcal{P}^{-}\right.$and $\left.P^{-} p H=H^{-}\right)$. It is clear that $p \in \mathcal{P}_{m}^{+} \Leftrightarrow p^{\perp} \in \mathcal{P}_{m}^{-}$. Let $r \in \mathcal{P}_{m}^{+}$. By the definitions (see Definitions 2.1 and 2.2 in $\left[5, \S 2\right.$, Chapter I]), the operator $J_{r}=r-r^{\perp}$ is a canonical symmetry with respect to the canonical decomposition $H=H_{r}^{+}[\dot{+}] H_{r}^{-}$, where $H_{r}^{+} \equiv r H$ and $H_{r}^{-} \equiv r^{\perp} H$. Let $\mathcal{A}$ be a $W^{*} J$-factor. Then $\mathcal{A}$ is a $W^{*} J$-factor of the same type in the Hilbert space $H$ with the inner product $(z, y)_{r} \equiv\left[J_{r} z, y\right]$.

With respect to the standard relations, an ordering $p \leq q \Leftrightarrow p q=q p=p$, the orthocomplementation $p \rightarrow p^{\perp} \equiv I-p$, and the orthogonal relation $p \perp q \Leftrightarrow p q=0$, the set $\mathcal{P}$ is a quantum logic. Any one-dimensional projection from $\mathcal{P}(B(H))$ can be represented in the form $p_{f}=[f, f][., f] f, f \in \Gamma$. Evidently, $p_{f}$ is an orthogonal projection $\Leftrightarrow f \in S \cap \Gamma\left(=S \cap\left(H^{+} \cup H^{-}\right)\right)$.

The logic $\mathcal{P}$ is not a $\sigma$-logic.

We will construct a sequence of mutually orthogonal projections $\left\{e_{n}\right\}_{1}^{\infty} \subset \mathcal{P}$ such that the supremum $\sum e_{n}$ does not exist in $\mathcal{P}$.

Let $H$ be such that $P^{+} H$ and $P^{-} H$ are infinite-dimensional separable Hilbert spaces. Let $\left\{\phi_{n}^{+}\right\}_{1}^{\infty},\left\{\phi_{n}^{-}\right\}_{1}^{\infty}$ be orthonormal bases in $P^{+} H, P^{-} H$. Put $f_{n} \equiv$ $(n+1)^{\frac{1}{2}} \phi_{n}^{+}+n^{\frac{1}{2}} \phi_{n}^{-}$. Then $\left[f_{n}, f_{n}\right]=\left(J f_{n}, f_{n}\right)=1, p_{f_{n}} \in \mathcal{P}^{+}(B(H))$, and $\left\{p_{f_{n}}\right\}_{1}^{\infty}$ is an orthogonal sequence. $\operatorname{lin}\left\{f_{n}, n \in N\right\}$ and $L=\overline{\operatorname{lin}\left\{f_{n}, n \in N\right\}}$ are positive 
linear subspaces ( see Definition 1.6 in $[5, \S 1$, Chapter I]). By [5, Theorem 4.7, $\S 4$, Chapter I $], L$ is a maximal positive subspace. Since $\left[f_{n}, f_{n}\right]\left(f_{n}, f_{n}\right)^{-1} \rightarrow 0$ by $[5$, Theorem $5.7, \S 5$, Chapter I], $L$ is not a Krein space, with respect to the product $[.,$.$] . This means that there is no projection p \in \mathcal{P}$ such that $p H=L$.

Assume now that the supremum $\sum_{1}^{\infty} p_{f_{n}} \in \mathcal{P}$ exists. Then $L \subseteq \sum_{1}^{\infty} p_{f_{n}} H$. Put

$$
p_{m} \equiv \sum_{1}^{m} p_{f_{n}}+\sum_{m+1}^{\infty}\left[\left(., \phi_{n}^{+}\right) \phi_{n}^{+}+\left(., \phi_{n}^{-}\right) \phi_{n}^{-}\right] \text {. }
$$

It is clear that $p_{m} \in \mathcal{P}, p_{m} \geq p_{m+1}, \forall m$, and $p_{m} \geq p_{f_{n}}, \forall m, n$. Hence $p_{m} \geq \sum_{1}^{\infty} p_{f_{n}}$, i.e. $p_{m} H \supseteq\left(\sum_{1}^{\infty} p_{f_{n}}\right) H, \forall m$, and $L=\cap_{1}^{\infty} p_{m} H \supseteq\left(\sum_{1}^{\infty} p_{f_{n}}\right) H$. Thus $L=\left(\sum_{1}^{\infty} p_{f_{n}}\right) H$. This is a contradiction.

Any sum $e=\sum e_{\iota}$, for $e_{\iota} \in \mathcal{P}, e_{\iota} \perp e_{j}(i \neq j)$ is said to be decomposition of $e$ ( the sum is understood in the strong sense ). A mapping $\mu: \mathcal{P} \rightarrow R$ is called a measure if $\mu(e)=\sum \mu\left(e_{\iota}\right)$ for every decomposition $e=\sum e_{\iota}$

Here, the convergence of an uncountable family of summands means that there exists only a countable set of nonzero terms in the family and the usual series with these summands converges absolutely.

A nonnegative measure $\mu$ is said to be a probability if $\mu(I)=1$. A measure $\mu$ is said to be indefinite if $\mu / \mathcal{P}^{+} \geq 0$ and $\mu / \mathcal{P}^{-} \leq 0$; semiconstant if there exist a faithful normal semifinite trace $\tau$ on $\mathcal{A}$ and a number $t$ such that $\mu(e)=t \tau\left(e_{+}\right)$, $\forall e \in \mathcal{P}$ or $\mu(e)=t \tau\left(e_{-}\right), \forall e \in \mathcal{P}$.

\section{The MAIN RESUlts}

Let $e, f \in \Pi$. We write $e \sim f$ if there exists a partial isometry $v \in \mathcal{A}$ with the initial projection $e$ and the final one $f$. We denote by $e_{p}^{+}$the orthogonal projection onto the subspace $P^{+} p H\left(=P^{+} e_{p} H\right), \forall p \in \mathcal{P}^{+}$.

Theorem 1. Any measure $\mu: \mathcal{P} \rightarrow R$ in the $W^{*} J$ - factor $\mathcal{A}$ is constant on the set of all maximal positive (negative) projections if and only if the measure $\mu$ is representable as a sum of semiconstant measures.

Proof. Let a measure $\mu$ be a sum of semiconstant measures. Then, obviously, $\mu$ is constant on the set of all maximal positive projections.

Conversely, let $\mu: \mathcal{P} \rightarrow R$ be constant on the set $\mathcal{P}_{m}^{+}$. Hence $\mu$ is constant on the set $\mathcal{P}_{m}^{-}$. For any $p \in \mathcal{P}^{+}$the projections $P^{+}$and $P^{+}-e_{p}^{+}+p$ are maximal positive. Hence

$$
\begin{gathered}
\mu\left(P^{+}-e_{p}^{+}\right)+\mu\left(e_{p}^{+}\right)=\mu\left(P^{+}\right) \\
=\mu\left(P^{+}-e_{p}^{+}+p\right)=\mu\left(\left(P^{+}-e_{p}^{+}\right)+\mu(p) .\right.
\end{gathered}
$$

Therefore,

$$
\mu\left(e_{p}^{+}\right)=\mu(p), \forall p \in \mathcal{P}^{+} .
$$

1. For the algebra $\mathcal{A}$ first assume that $\mathcal{A}=B(H)$. If $\operatorname{dim} H=2$, then Theorem 1 is trivial. Now assume that $\operatorname{dim} H>2$. For any vector $z \in S \cap H^{-}$we set $L_{z}^{-}=\left\{p_{f} \in \mathcal{P}:\left(P^{-}-p_{z}\right) f=0\right\}$. In a similar way, for any vector $y \in S \cap H^{+}$we can set $L_{y}^{+}=\left\{p_{f} \in \mathcal{P}:\left(P^{+}-p_{y}\right) f=0\right\}$. The equality (2) gives that $\mu \equiv$ const 
$\left(\equiv c_{+}(z)\right)$ on the set $L_{y}^{+} \cap \mathcal{P}^{+}$and $\mu \equiv$ const $\left(\equiv c_{-}(z)\right)$ on the set $L_{z}^{-} \cap \mathcal{P}^{-}$. Now let the vector $r \in S \cap H^{-}$be such that $(r, z) \neq 0$ and $p_{r} \neq p_{z}$. We can assume that $(r, z) \in R \backslash\{0\}$. Choose a negative vector $f \in \operatorname{lin}_{R}\{r, z, y\}$ such that $p_{f} \in L_{r}^{-}$ and the real line $\operatorname{lin}_{R}\{f, z\}$ contains at least one positive vector. Then there exists a positive vector $g \in \operatorname{lin}_{R}\{f, z\}$ such that $p_{g} \in L_{y}^{+}$and $\left(P^{-} g, z\right)=0$. This means that $p_{g} p_{z}=p_{z} p_{g}=0$. Then $p_{z}+p_{g} \in \mathcal{P}$ and $p_{z}+p_{g}-p_{f} \in L_{y}^{+}$. We have

$$
\begin{gathered}
c_{+}(y)=\mu\left(p_{z}+p_{g}-p_{f}\right)=\mu\left(p_{z}\right)+\mu\left(p_{g}\right)-\mu\left(p_{f}\right) \\
=c_{-}(z)+c_{+}(y)-c_{-}(r) .
\end{gathered}
$$

Hence $c_{-}(z)=c_{-}(r)$ and, therefore, the function $z \rightarrow c_{-}(z), z \in S \cap H^{-}$, is constant. Similarly, the function $y \rightarrow c_{+}(y), y \in S \cap H^{+}$, is constant, too. Thus the measure $\mu$ is constant $\equiv c_{+}\left(\equiv c_{-}\right)$on the set of all one-dimensional positive (negative) projections. This means that

$$
\mu(e)=c_{+} \operatorname{tr}\left(e_{+}\right)+c_{-} \operatorname{tr}\left(e_{-}\right), \forall e \in \mathcal{P}, \text { where }(0 \cdot \infty \equiv 0) .
$$

2. Now let $\mathcal{A}$ be a continuous $W^{*} J$-factor. Choose $e$ and $f \in \Pi$ such that $e+f \leq P^{+}$and $e \sim f$. Let $v \in \mathcal{A}$ be a partial isometry with the initial projection $e$ and the final one $f$. Assume there is a partial isometry $w \in \mathcal{A}$ with the initial projection $e$ and the final one $w w^{*} \leq P^{-}$. The minimal *-algebra $\mathcal{A}(e, w, v)$ generated by the operators $e, v, w$ is a $W^{*} J$-factor of type $I_{3}$ (acting in the Hilbert space $\left(e+p+v v^{*}\right) H$ with the canonical symmetry $\left.J^{\prime}=e+f-v v^{*}\right)$. For any positive projection $p$ maximal in $\mathcal{A}(e, w, v)$, we have $p+P^{+}-e-f \in \mathcal{P}_{m}^{+}$. Because $\mu$ is constant on $\mathcal{P}_{m}^{+}$, it follows that $\mu$ is also constant $(=\mu(e)+\mu(f))$ on the set of all maximal in $\mathcal{A}(e, w, v)$ positive projections. By Section $1, \mu(e)=\mu(f)$. Therefore, if the projection $P^{+}$is an infinite projection with respect to $\mathcal{A}$, then $\mu(e)=0$ for any projection $e \in \mathcal{P}, e \leq P^{+}$. Due to the equality (2) we conclude that $\mu / \mathcal{P}^{+}$identically equals zero. In a similar way, if the projection $P^{-}$is infinite then $\mu / \mathcal{P}^{-} \equiv 0$.

Now let the projection $P^{+}$be a finite one, with respect to $\mathcal{A}$. Let $e, p \in \mathcal{P} \cap \Pi$, $e \vee p \leq P^{+}$and $e \sim p$. From the continuity of the factor $\mathcal{A}$ it follows that there exist decompositions $e=e_{1}+e_{2}$ and $p=p_{1}+p_{2}$, where $e_{1}$ and $p_{1}$ are projections from $\mathcal{A}$ such that $e_{\iota} \perp p_{\iota}$ and $e_{\iota} \sim p_{\iota}(\iota=1,2)$. Again, by the step $1, \mu\left(e_{\iota}\right)=\mu\left(p_{\iota}\right)$. Hence $\mu(e)=\mu(p)$. Therefore, the restriction of $\mu$ to $\left\{e \in \mathcal{P}: e \leq P^{+}\right\}$is unitary invariant (i.e., $e \sim f \Rightarrow \mu(e)=\mu(f))$. Let $\tau$ be a faithful normal semifinite trace on $\mathcal{A}$. Then there exists a unique constant $c \in R$ such that $\mu(p)=\mu\left(e_{p}\right)=c \tau(p)$, $\forall p \in \mathcal{P}^{+}$.

By analogy, if the projection $P^{-}$is finite then there exists a constant $t \in R$ such that $\mu(p)=t \tau(p), \forall p \in \mathcal{P}^{-}$.

In the general case, $\mu(p)=c \tau\left(p_{+}\right)+t \tau\left(p_{-}\right), \forall p \in \mathcal{P}(0 \infty \equiv 0)$.

A $W^{*} J$-factor $\mathcal{A}$ is said to be a $W^{*} P$-factor if at least one of the projections $P^{+}$ or $P^{-}$is finite with respect to $\mathcal{A}$.

Theorem 2. Let $\mu: \mathcal{P} \rightarrow R^{+}$be a probability measure on a $W^{*} J$-factor $\mathcal{A}$ (the type of $\mathcal{A}$ is different from $\left.I_{2}\right)$. Then $\mathcal{A}$ is a $W^{*} P$-factor and $\mu$ is a sum of semiconstant measures.

Proof. Let $\mu$ be a nonnegative measure. The proof will consist of several steps. 
1) Suppose first $\mathcal{A}=B(H)$, where $3 \leq \operatorname{dim} H<\infty$. The function $\nu(p) \equiv$ $\operatorname{tr}(\mu(I) J p)-\mu(p), \forall p \in \mathcal{P}$ is an indefinite measure on $\mathcal{P}$ because if $p \in \mathcal{P}^{+}(p \neq 0)$ then

$$
\nu(p)=\mu(I) \operatorname{tr}(p J)-\mu(p) \geq \mu(I) \operatorname{tr}\left(e_{p}\right)-\mu(p) \geq \mu(I)-\mu(p) \geq 0,
$$

and if $p \in \mathcal{P}^{-}(p \neq 0)$ then $\nu(p) \leq-\mu(I)-\mu(p) \leq 0$. By Theorem [4], there exist a $J$-selfadjoint operator $T$ (i.e., $T=J T^{*} J$ ) and a number $c$ such that $\nu(p)=$ $\operatorname{tr}(T p)-\operatorname{cdim}\left(p_{+} H\right)$. Hence $\mu(p)=\operatorname{tr}((\mu(I) J-T) p)+\operatorname{cdim}\left(p_{+} H\right), \forall p \in \mathcal{P}$. It is obvious that $\mu(p)=\operatorname{tr}((\mu(I) J-T+c I) p)-\operatorname{cdim}\left(p_{-} H\right), \forall p \in \mathcal{P}$.

Let $z \in H^{+} \cap S, y \in H^{-} \cap S$ and $v=(., z) y$. Put $B \equiv \mu(I) J-T$. Since $B=J B^{*} J$, we get

$$
\begin{gathered}
(B z, y)=(B J z, y)=\left(J B^{*} z, y\right)=\left(B^{*} z, J y\right) \\
=-\left(B^{*} z, y\right)=-(z, B y)=-\overline{(B y, z)} .
\end{gathered}
$$

Hence

$$
\begin{gathered}
0 \leq \mu\left(p\left(\beta p_{z}, e^{\iota \theta} v\right)\right)=\operatorname{tr}\left(B p\left(\beta p_{z}, e^{\iota \theta} v\right)\right)+c \\
=\beta \operatorname{tr}\left(B p_{z}\right)+\left(\beta^{2}-\beta\right)^{1 / 2}\left(\operatorname{tr}\left(e^{\iota \theta} B v\right)-\operatorname{tr}\left(e^{-\iota \theta} B v^{*}\right)\right)-(\beta-1) \operatorname{tr}\left(B p_{y}\right)+c \\
=\beta(B z, z)+\left(\beta^{2}-\beta\right)^{1 / 2}\left(e^{\iota \theta}(B y, z)-e^{-\iota \theta}(B z, y)\right)-(\beta-1)(B y, y)+c \\
=\beta((B z, z)-(B y, y))+2\left(\beta^{2}-\beta\right)^{1 / 2} \operatorname{Re}\left(e^{\iota \theta}(B y, z)\right)+(B z, z)+c \leq 1, \\
\forall \beta>1, \forall \theta \in R .
\end{gathered}
$$

Therefore, $(B z, z)=(B y, y)$ and $(B y, z)=0, \forall z \in H^{+} \cap S, \forall y \in H^{-} \cap S$. Hence $B=a I$, where $a \equiv(B z, z)$. Therefore,

$$
\begin{aligned}
\mu(p) & =\operatorname{tr}(B p)+c \operatorname{dim}\left(p_{+} H\right)=\operatorname{atr}(p)+c d i m\left(p_{+} H\right) \\
& =(a+c) \operatorname{dim}\left(p_{+} H\right)+\operatorname{adim}\left(p_{-} H\right), \forall p \in \mathcal{P} .
\end{aligned}
$$

Thus $\mu$ is a sum of semiconstant measures.

2) Let $\operatorname{dim} H=\infty$. It follows from the step 1) that $\mu\left(p_{y}\right) \equiv$ const $(=a \geq 0)$ $\left\{\mu\left(p_{z}\right) \equiv\right.$ const $\left.(=c+a \geq 0)\right\}$ on the set of all one-dimensional orthogonal negative \{positive projections. It is clear that if $\operatorname{dim} H^{-}=\infty$ then $a=0$. This means that $c d i m H^{+}=\mu\left(P^{+}\right)=\mu(I)<\infty$. Hence $\operatorname{dim} H^{+}<\infty$ and $\mu(p)=c \operatorname{dim} p_{+} H, \forall p \in$ $\mathcal{P}$. By analogy if $\operatorname{dim} H^{+}=\infty$, then $a+c=0, \operatorname{dim} H^{-}<\infty$ and $\mu(p)=a d i m p_{-} H$, $\forall p \in \mathcal{P}$.

3) Now let $\mathcal{A}$ be a continuous $W^{*} J$-factor. By analogy with the proof of Theorem 1 and from the step 1), we have

$$
e \sim f \Rightarrow \mu(e)=\mu(f)
$$

where $e, f \leq P^{+}$or $e, f \leq P^{-}$. Let $r \in \mathcal{P}_{m}^{+}$. Then $\mathcal{A}$ is a continuous $W^{*} J$-factor in $H$ with the Hilbert product $\left[J_{r} .,.\right]$. By analogy, if $e, f \in \mathcal{P}$ and $e, f \leq r$ or $e, f \leq r^{\perp}$, and, in addition, $e \sim f$ with respect to the product $\left[J_{r} .,.\right]$ then $\mu(e)=\mu(f)$. 
a) Assume that $P^{+}$is the infinite projection with respect to $\mathcal{A}$. Then every $p \in \mathcal{P}_{m}^{+}$is infinite, too. We can represent $P^{+}$as $P^{+}=\sum_{i=1}^{\infty} e_{i}$, where $\forall e_{i} \in \mathcal{P}^{+}$ and $e_{i} \sim e_{j}(i \neq j)$. By (3),

$$
0 \leq \mu\left(P^{+}\right)=\sum_{i=1}^{\infty} \mu\left(e_{i}\right)=+\infty \mu\left(e_{1}\right) \leq \mu(I)<+\infty .
$$

Hence $\mu\left(P^{+}\right)=0$. It follows that $\mu / \mathcal{P}_{m}^{+} \equiv 0$. By Theorem $1, \mu$ is a sum of semiconstant measures.

b) Now, assume that $P^{+}$and $P^{-}$are finite with respect to $\mathcal{A}$. Let $r \in \mathcal{P}_{m}^{+}$. We can write $r=p+q+g$, where $p, q, g \in \mathcal{P}^{+}$and $p \sim q \sim g$ in $H$ with the Hilbert product $\left[J_{r} .,.\right]$. Denote by $p_{i}$ the orthogonal projection onto $H \ominus\{x, p x=0\}$. Then $e_{p} \sim p_{i}$. By the choice of $p$, there exists a decomposition $P^{+}=e_{p}^{+}+e+f$, where $e, f \in \mathcal{P}$ and $e_{p}^{+} \sim e \sim f$, such that $e \perp p_{i}$. Then $e+p \in \mathcal{P}^{+}$. There exists $d \in \mathcal{P}_{m}^{+}$ such that $e+p \leq d$. Because of $e \sim e_{p}^{+}$and $e_{p}^{+} \sim e_{p}$, we have $e \sim p$ in $H$ with the Hilbert product $\left[J_{d} .,.\right]$. By $(3), \mu(p)=\mu(e)$. Since $\mu(e)=\mu\left(e_{p}^{+}\right)$, it follows that $\mu(p)=\mu\left(e_{p}^{+}\right)$. Hence

$$
\mu\left(P^{+}\right)=3 \mu\left(e_{p}^{+}\right)=3 \mu(p)=\mu(p+q+g)=\mu(r) .
$$

Thus $\mu / \mathcal{P}_{m}^{+} \equiv$ const. Again, by Theorem 1, $\mu$ is a sum of semiconstant measures.

\section{REFERENCES}

1. G. Mackey, The mathematical foundations of quantum mechanics, Benjamin, New York, 1963. MR 27:5501

2. A. M. Gleason, Measures on closed subspaces of a Hilbert space, J. Math. Mech 6 (1957), 885-893. MR 20:2609

3. K. Yu. Dadashyan and S.S. Horujy, On Field algebras in quantum theory with indefinite metric, Theor. and Math. Phys. 54 (1983), 57-77 (Russian), English transl. in Theor. and Math. Phys. 54 (1983), 35-48. MR 85d:81082

4. M. S. Matvejchuk, Measure on quantum logics of subspaces of a J-space, Sibirskii Matematicheskii Zhurnal 32 (1991), 101-112 (Russian); English transl. in Siberian Mathem. J. 32 (1991), 265-272. MR 92j:46137

5. T. Ya. Azizov and I. S. Iokhvidov, Linear operators in space with an indefinite metric, Nauka, Moscow. 1986 (Russian); English transl. Wiley, New York, 1989. MR 90j:47042

6. M. S. Matvejchuk, A description of indefinite measures in $W^{*} J$-factors, Dokl. Akad. Nauk SSSR 319 (1991), 558-561; English transl. in Soviet Math. Dokl. 44 (1992), 161-165. MR 93a: 46123

Department of Mechanics and Mathematics, Kazan State University, 18 Lenin St., 420008, KaZAN, Russia

E-mail address: marjan@niimm.kazan.su 